\title{
Bump-Induced Transition in Compressible High Reynolds Number Flow: Experimental Results and Correlation with Linear Stability Analysis
}

Introduction Surface bumps can occur on aircraft components (e.g., at or between structural joints, ribs and stringers) and can induce a marked amplification of boundary-layer instabilities, thus leading to premature transition to turbulence. The influence of bumps on boundary-layer transition was systematically studied in combination with the effect of variations in the following parameters: streamwise (global) pressure gradient, freestream Mach number (up to $\mathbf{M}=\mathbf{0 . 7 7}$ ) and chord Reynolds number (up to $\mathbf{R e}=\mathbf{1 0} \cdot \mathbf{1 0}^{6}$ ). The experimental investigations were conducted in a (quasi-) two-dimensional flow in the Cryogenic Ludwieg-Tube Göttingen (DNW-KRG).

\section{Wind-tunnel model BuLASTra}

$500 \mathrm{~mm}$

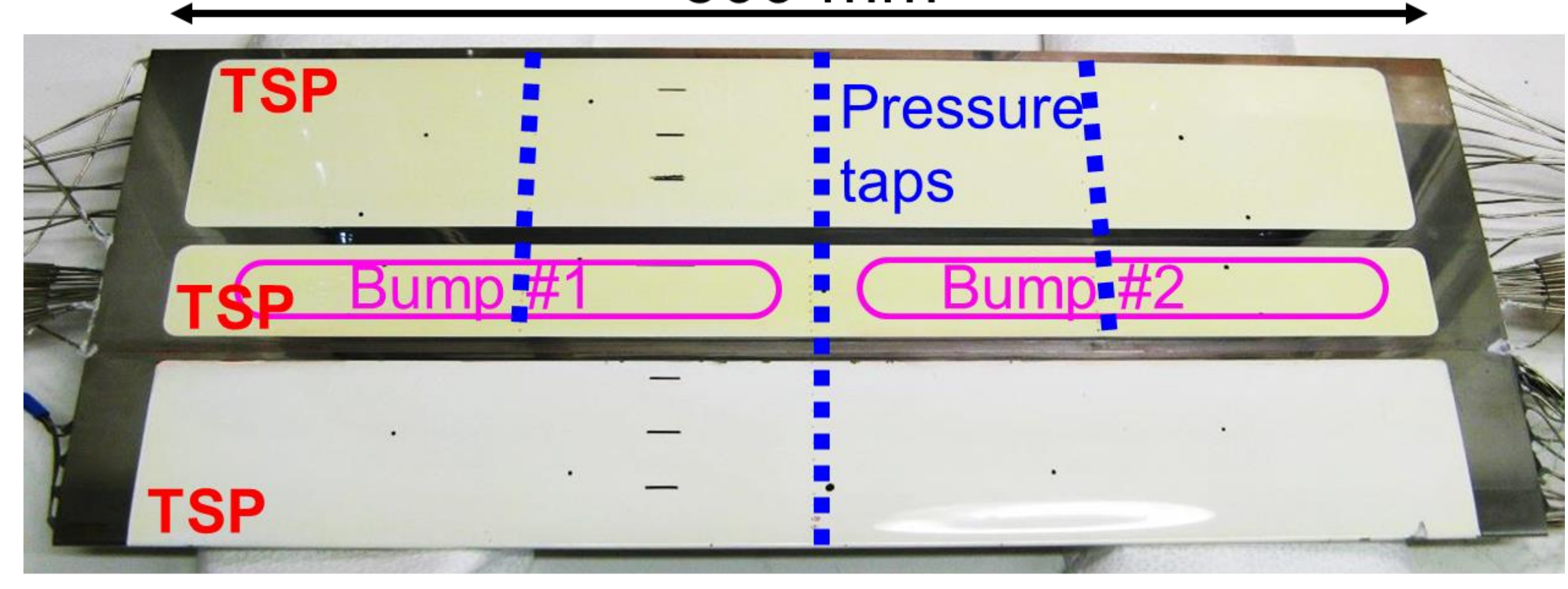

Leading edge at the bottom of the image

Model chord $c=\mathbf{2 0 0} \mathbf{~ m m}$, bump crest at $x / c=45 \%$

Temperature-Sensitive Paint (TSP) for transition detection

\section{Bump shape}

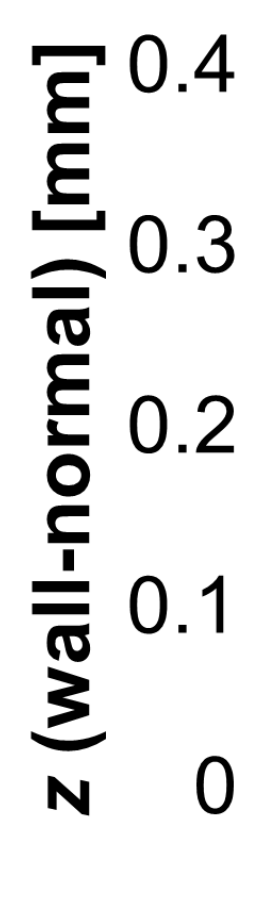

Three sinusoidal bumps

(quasi-2D) investigated

\section{Numerical setup}

- Laminar basic flow computed with boundary-layer code cOCO using the experimental pressure distributions and considering an isothermal wall.

- Approach applicable only for cases without separation, i.e. mostly the reference and small bump configurations.

- Compressible linear local stability analyses (LST) using both LILO and NOLOT.

\section{Effect of the variation of the streamwise (global) pressure gradient (Hartree parameter $\boldsymbol{\beta}_{\mathrm{H}}$ )}

Experimental results (TSP)

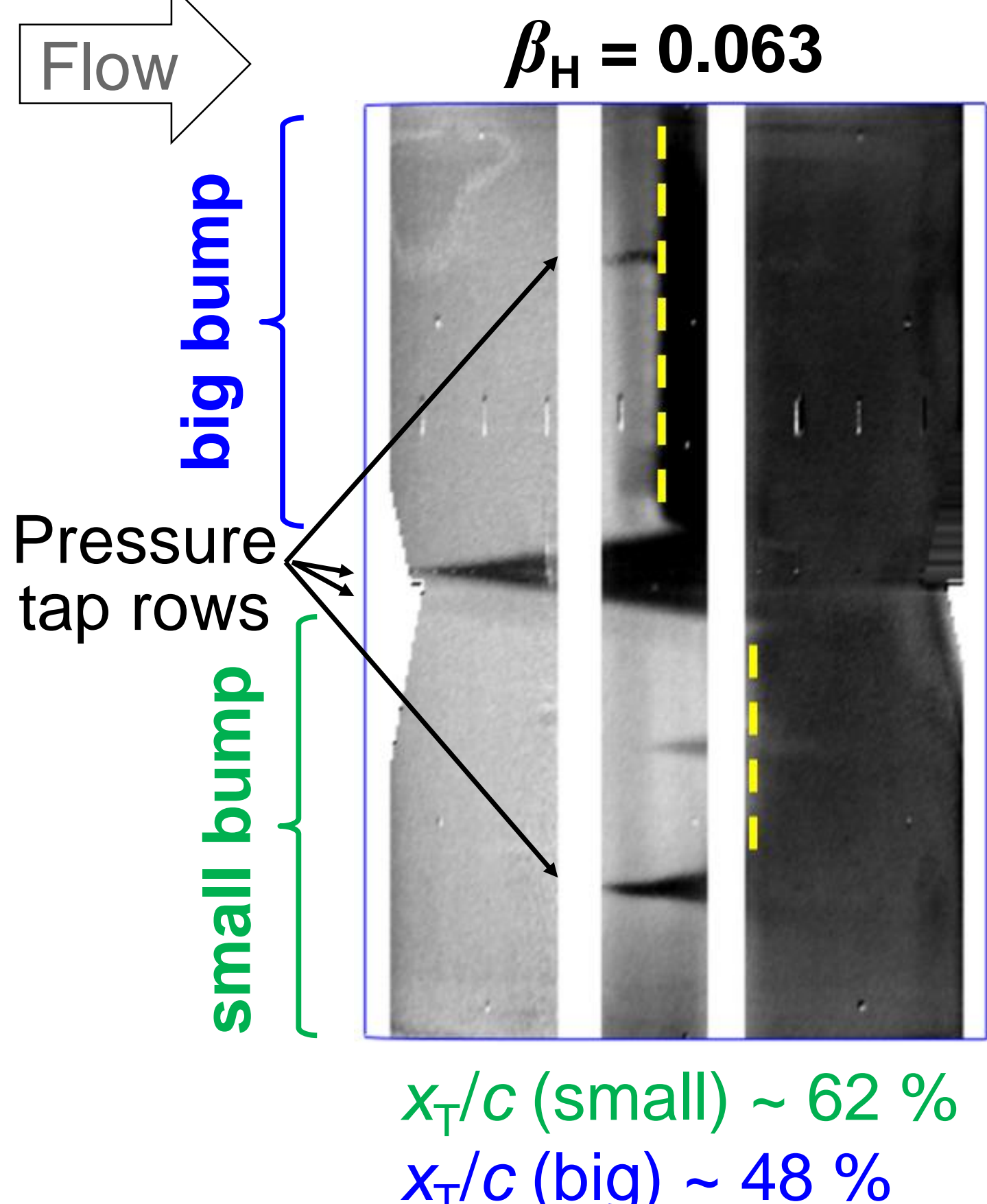

Transition location $x_{\mathrm{T}} / \boldsymbol{c}$ shown by dashed yellow lines

- Boundary-layer transition location moved upstream with increasing bump height.

- In the case of the small bump, transition was very sensitive to the global pressure gradient $\beta_{\mathrm{H}}$.

- With the mid bump, the transition sensitivity on $\beta_{\mathrm{H}}$ depended on Mach and Reynolds numbers.

- Transition induced by the big bump was essentially unaffected by $\beta_{\mathrm{H}}$
Numerical results ( $N$-factors)

small bump, $\boldsymbol{\beta}_{\mathrm{H}}=\mathbf{0 . 0 6 3}$

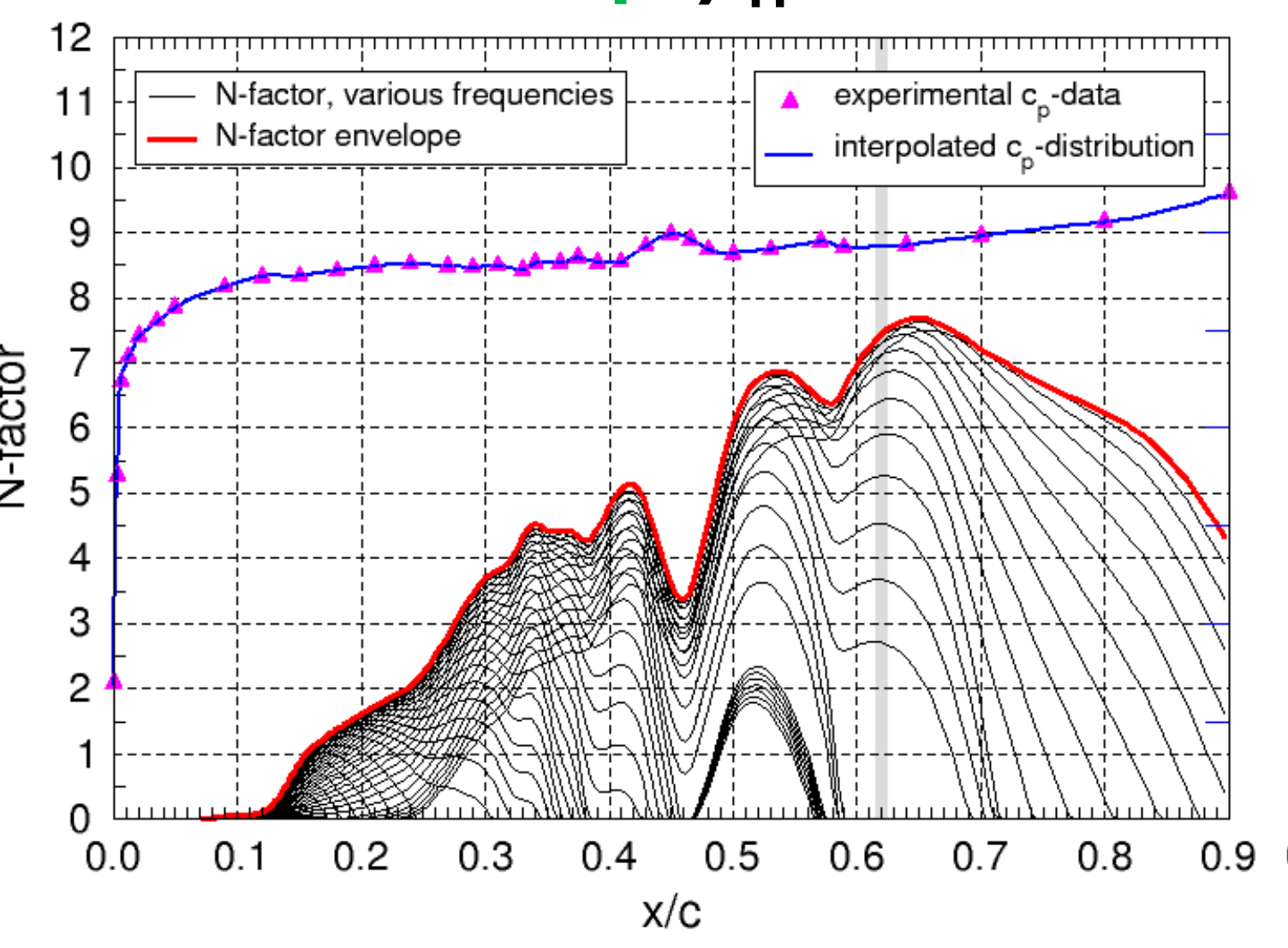

small bump, $\boldsymbol{\beta}_{\mathrm{H}}=\mathbf{0 . 0 9 6}$

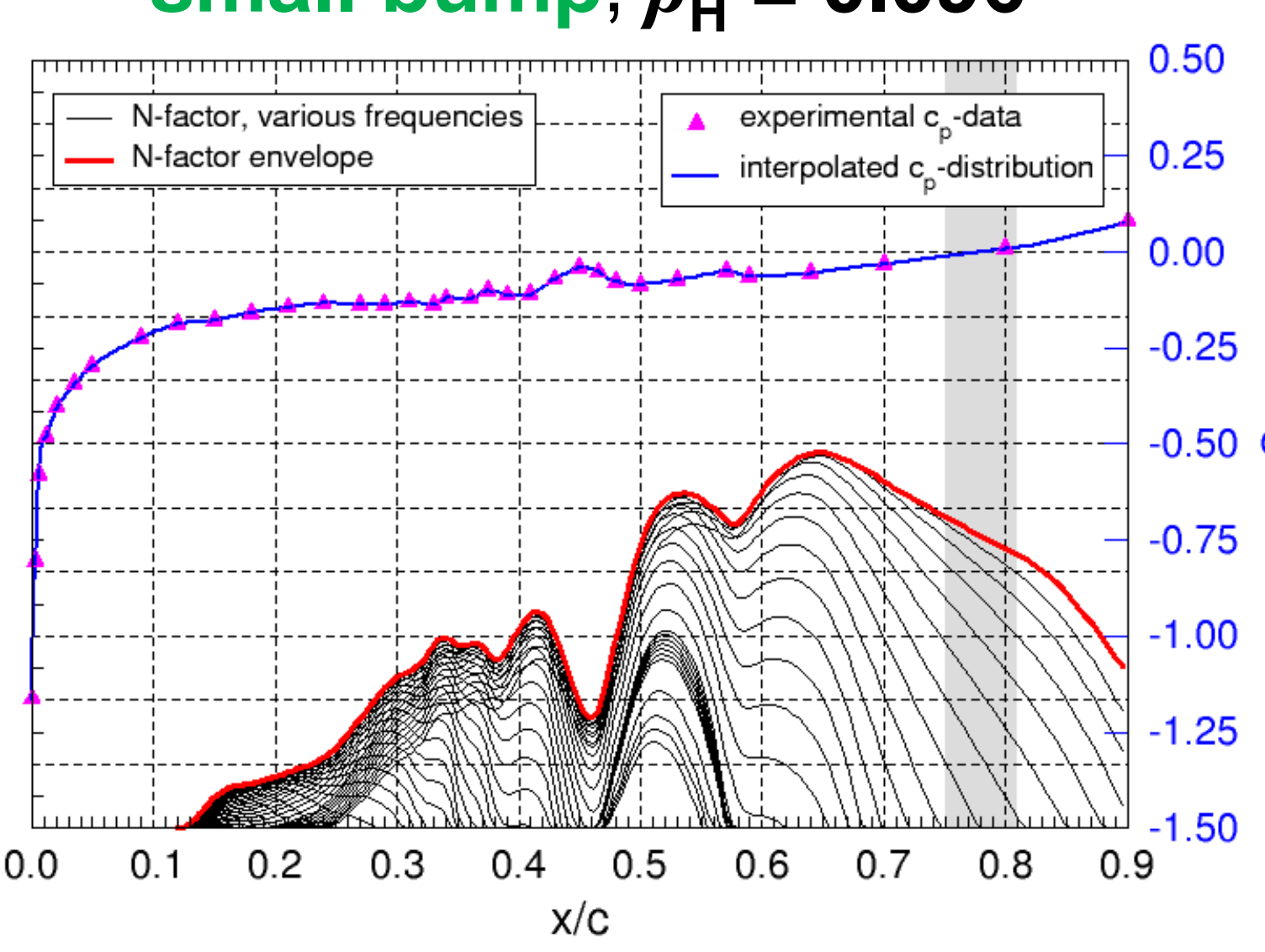

Results of LST obtained using NOLOT

Transition location $\boldsymbol{X}_{\mathrm{T}} / \boldsymbol{c}$ and its uncertainty shown by gray bars

- Correlation of the experimental data with results from LST for the small bump configuration:

- Transition $\mathrm{N}$-factors $\mathrm{N}_{\mathrm{T}}$ decreased with increasing $\mathrm{M}$ and increasing $\beta_{\mathrm{H}}$.

- The decrease of $N_{T}$ with increasing $M$ may be related to the increasing level of total pressure fluctuations in DNW-KRG.

- In some cases, the transition locations found in the experiments could not be explained via the adopted $e^{\mathrm{N}}$-methodology. 